\title{
Fluorescent and Electron Microscopy Revealed Critical Roles of C2cd3 in Centriolar Distal Appendage Assembly and Cilia Biogenesis
}

\author{
Xuan $\mathrm{Ye}^{1}$, Huiqing Zeng ${ }^{1}$, Gang Ning ${ }^{2}$ and $\underline{\text { Aimin } \mathrm{Liu}^{3}}$ \\ 1. Department of Biology, Eberly College of Science, The Pennsylvania State University, University \\ Park, USA. \\ 2. Microscopy and Cytometry Facility, The Huck Institutes of the Life Sciences, The Pennsylvania State \\ University. University Park, USA \\ 3. Department of Biology, Eberly College of Science, Center for Cellular Dynamics, The Huck Institutes \\ of the Life Sciences, The Pennsylvania State University, University Park, USA
}

The primary cilium is a hair-like protrusion present on the surface of almost all vertebrate cells and is crucial for development and physiology [1]. Recent proteomic and genomic studies suggested that more than 3500 proteins are involved in cilia biogenesis and function; however, the mechanisms underlying their functions remain poorly understood.

In a previous study, we identified $\mathrm{C} 2$ domain containing protein $3(\mathrm{C} 2 \mathrm{~cd} 3)$ as an essential regulator of primary cilium biogenesis in mouse, and found that a fluorescently-tagged $\mathrm{C} 2 \mathrm{~cd} 3$ protein localized to the base of the cilium [2]. To better determine the localization and function of this new regulator of cilia formation, we generated a polyclonal antibody specifically recognizing $\mathrm{C} 2 \mathrm{~cd} 3$ protein. Through immunofluorescence analysis, we identified a dynamic pattern of $\mathrm{C} 2 \mathrm{~cd} 3$ subcellular localization. In interphase, $\mathrm{C} 2 \mathrm{~cd} 3$ localizes to granules around the ciliary basal, and we determined that these granules are centriolar satellites, a structure involved in cilium formation. During mitosis, C2cd3 specifically localizes to two find dots at each spindle pole, suggesting that it may be a centriole protein.

Centrioles are tiny cylindrical structures surrounded by bundles of microtubules [3]. Each centrosome or spindle pole comprises two centrioles and surrounding pericentriolar matrix. These two centrioles are synthesized at different time points. The one synthesized in the previous cell cycle is called mother centriole, whereas the one synthesized in the current cell cycle is called the daughter centriole. The mother centriole acquires decorations on its side called the subdistal appendages and distal appendages (Fig. 1A). We observed the localization of $\mathrm{C} 2 \mathrm{~cd} 3$ using a fluorescently-labeled antibody and a high-resolution confocal microscope, and found that $\mathrm{C} 2 \mathrm{~cd} 3$ localized to the distal end of both mother and daughter centrioles. In particular, it localizes to the base of distal appendages on the mother centriole (Fig. 1A).

The localization of $\mathrm{C} 2 \mathrm{~cd} 3$ at the base of the distal appendages prompted us to examine its role in assembling these appendages. By immunofluorescent analysis we found that five known components of the distal appendages, Sclt1, Ccdc41, Cep89, Fbf1 and Cep164, failed to localize to the mother centriole, suggesting that $\mathrm{C} 2 \mathrm{~cd} 3$ is essential for the assembly of the distal appendages (Fig. 1B).

Distal appendages are important for a series molecular events leading to the formation of a cilium. These include the recruitment of Tau tubulin kinase 2 (Ttbk2), which is important for the removal of an inhibitor of cilium formation, Centriolar Protein of 110kD (Cp110) [4]. Finally, various intraflagellar transport (Ift) proteins essential for cargo transport inside the cilium, are recruited to the distal 
appendages. Consistent with the failure in distal appendage assembly, Ttbk2, Ift 88 and Ift52 are not recruited to, and $\mathrm{Cp} 110$ is not removed from, the mother centriole, in the absence of C2cd3.

The first step in cilium formation involves the docking of Golgi-derived ciliary vesicles to the distal appendages of the mother centriole to convert it to the ciliary basal body $[1,4]$. To determine whether this important step was affected, we examined a fluorescently-labeled ciliary vesicle marker Smoothened (Smo). We found that Smo-containing vesicles are at the mother centriole of the wild type, but not the $C 2 c d 3$ mutant cells (Fig. 1B). To further confirm this result, we examined ultra-thin sections of developing embryos with transmission electron microscope, and found that nearly half of the centrioles in wild type embryos were associated with vesicles, whereas no centriole in C2cd3 mutant embryos was. These results suggest that $\mathrm{C} 2 \mathrm{~cd} 3$ is crucial for the docking of vesicles to the mother centriole.

In conclusion, our optical and electron microscopy analyses reveal that failure in the assembly of distal appendages, the subsequent recruitment of ciliogenic proteins and docking of vesicles, underlie the defects in cilium formation in the absence of $\mathrm{C} 2 \mathrm{~cd} 3$ [5].

\section{References:}

[1] JM Gerdes, EE Davis, N Katsanis, Cell 137 (2009), p. 32.

[2] AN Hoover et al, Development 135 (2008), p4049.

[3] M Bornens, Science 335 (2012), p422.

[4] BE Tanos et al, Genes Dev. 27 (2013), p163.

[5] The authors acknowledge Drs Kathryn Anderson, Tamara Caspary, James Sillibourne, Andreas Merdes, Joseph Gleeson, Philip Beachy for reagents. American Heart Association (\# 0830174N) and Pennsylvania State University provided funding for this work.

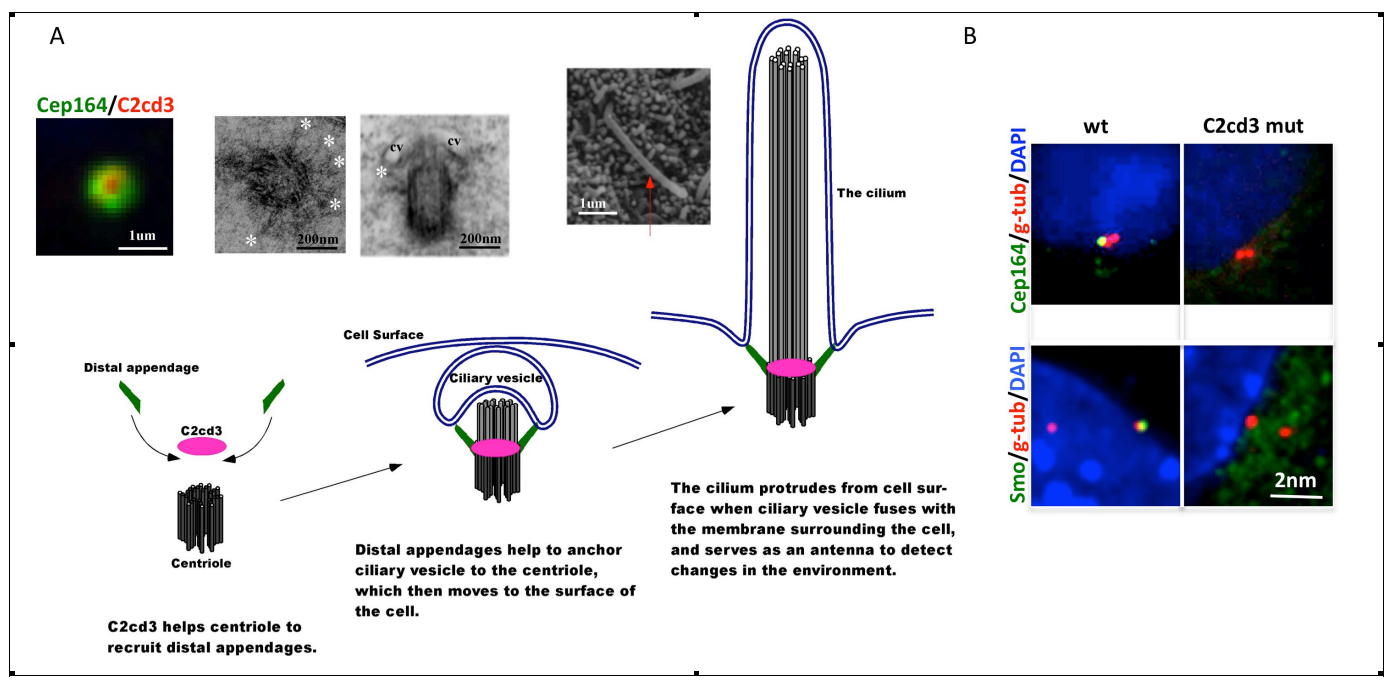

Figure 1. C $2 \mathrm{~cd} 3$ localizes to the distal end of the centrioles and regulates distal appendage assembly and vesicle docking. (A) Asterisks point to distal appendages. cv indicates ciliary vesicle. Red arrow points to a node cilium. (B) The distal appendages (Cep164) and ciliary vesicles (Smo) are not recruited to the mother centriole (g-tub). 